\title{
Determination of the Near Wall Flow of a Multi-Stage Tesla valve Using PIV and PTV
}

\section{Yeganeh Saffar, Shadi Ansari, Reza Azadi, Jan Raffel, David S. Nobes* and Reza Sabbagh}

\author{
Department of Mechanical Engineering, University of Alberta, Canada \\ *dnobes@ualberta.ca
}

\begin{abstract}
Particle image velocimetry (PIV) and particle tracking velocimetry (PTV) are two popular methods to measure the velocity in complex geometries such as the Tesla valve. This paper provides an investigation on the application of a tessellation meshing method for interpolating non-uniform velocity vectors calculated using PTV. The procedure to apply this method containing mask generation and mesh study is described. The results are compared to the PIV results particularly where the near wall results are important. The result of the flow field calculated by the application of the tessellation method on the PTV results are presented for a two-stage Tesla valve operated in the range of $\mathrm{Re}=100$ to 600 both in forward and reverse configuration.
\end{abstract}

\section{INTRODUCTION}

The Tesla valve is a nonmoving mechanical flow control device used to restrict the flow preferentially in one direction Tesla (1920). Its geometry generates a higher pressure drop in the flow in one direction and a lower pressure drop when it is used in the reverse direction. The simplicity of its fabrication and the operation in comparison to the conventional valves makes it an appropriate choice for different applications Porwal et al. (2018), Wahidi et al.(2020). The efficiency of the Tesla valve depends on the pressure drop of the flow in each direction which is related to the flow field inside the channel (Gamboa et al. (2005). Therefore, understanding the internal flow can lead to a better insight into the design and optimization of the Tesla valve J. Raffel (2019).

Particle tracking velocimetry (PTV) is a non-intrusive imaged based velocimetry method that follows a particle in two successive frames of images captured of the flow. In the cases of high velocity gradients, PTV provides a higher spatial resolution which is an advantage in comparison to particle image velocimetry (PIV) M. Raffel et al. (2007). These two methods are great candidates to study the flow field in mini and micro channels such as the Tesla valve S. Ansari et al. (2018) and J. Raffel et al. (2021). However, both of these methods need additional corrections based on the geometry of the domain and the flow field conditions. Unlike PIV, PTV is highly dependent on the number of the seeding particles and their distribution in the flow field. This increases the possibility of having missing velocity values in regions with fewer particles such as the near wall region M. Raffel et al. (2007).

In this work, the flow behavior inside a Tesla valve is studied applying PIV and PTV and a method is proposed to improve the quality of results. The determination of the near wall flow characteristics in this complex channel flow geometry is affected by the type of particle analysis carried out to obtain the flow velocity. Thus, this research focuses on how best to determine the near wall characteristics. The use of a tessellation meshing method to discretize the domain and assimilate the experimental data to calculate velocity in the wall region is discussed. 


\section{EXPERIMENTAL SETUP}

A transparent flow channel shown in Figure 1, was fabricated using a 3D printer and a clear resin material. The top window is a laser cut acrylic sheet chosen to give optical access to the channel. The channel width, $d=2 \mathrm{~mm}$ and a two-stage Tesla valve is used for this experiment. To ensure that a uniform flow is entering the Tesla valve, a honeycomb is designed before each forward and reverse inlets. The same cell is used for both forward and reverse flow directions, by changing the direction of the flow stream.

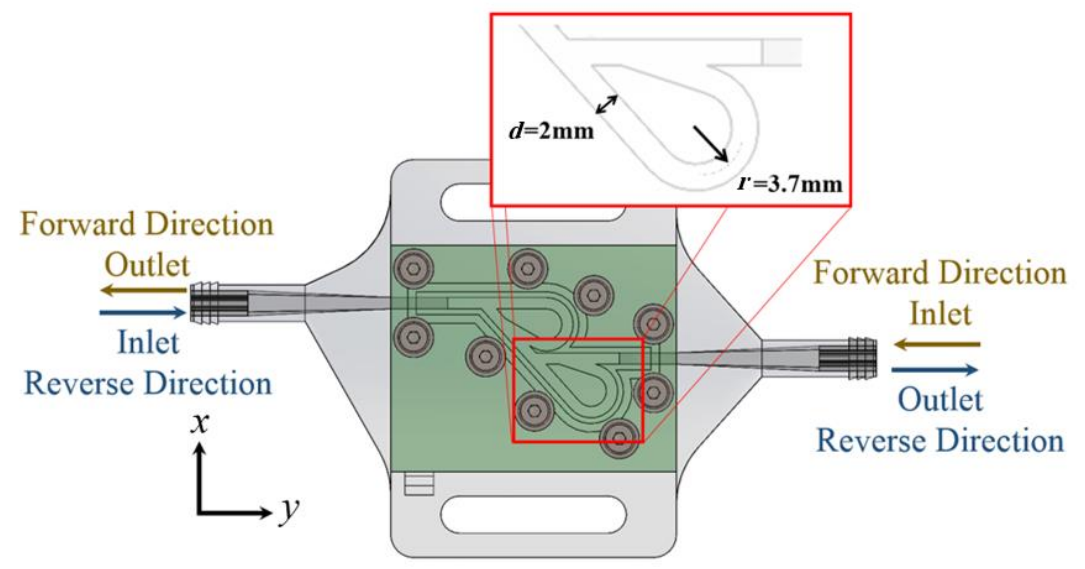

Figure 1: The solid model of the Tesla valve and the inlet and outlet of the flow stream for both forward and reverse flow direction

A schematic of the experimental imaging setup is shown in Figure 2 which is used to image tracer particles inside the Tesla valve with the particle shadow velocimetry (PSV) technique. A high-speed CCD camera is used to capture images for Reynolds numbers between $100 \leq \mathrm{Re} \leq 300$ with $2000 \mathrm{fps}$ and the resolution of 1536 pixels $\times 768$ pixels. For conditions of Reynolds numbers of $400 \leq \operatorname{Re} \leq 600,4000 \mathrm{fps}$ and the resolution of 1280 pixels $\times 512$ pixels is used. A green LED is used to illuminate the channel from below to visualize the motion of the particles in a shadowgraph configuration. Inside the channel, a mixture of water and glass bead particles with a different diameter of $10 \mu \mathrm{m}$ and $20 \mu \mathrm{m}$ (Dynoseeds TS10, TS 20) for each experiment is used.

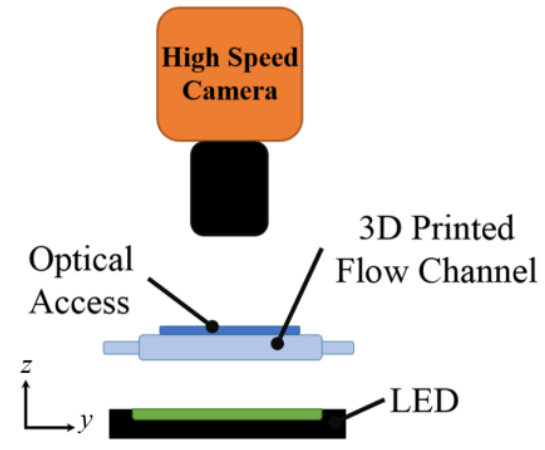

Figure 2: Schematic of the optical setup showing shadowgraph configuration 


\section{Image Processing}

Images of the flow are processed to obtain the velocity vectors in the flow field. This is undertaken by applying the tessellation meshing method before processing the images for PIV/PTV. To generate a mesh for the tessellation, obtaining the boundary of the geometry is required. This provides the ability to distribute the nodes of the mesh on the channel walls. The raw images, however, need to be masked to eliminate unwanted shadow regions and their effect on image analysis. Since this specific geometry cannot be simply generated using mathematical approaches, raw images of the flow field are alternative references that can be used in the masking process. The raw image shown in Figure 3(a) is an instance which shows the geometry of the channel with seeding particles and other extra shadows. All of these additional shadows increase the difficulty in finding the channel walls.

To overcome this problem, for each Reynolds number, 1000 instantaneous frames containing flow and tracer particles are used to calculate the standard deviation of the images intensity. Figure 3(b) shows the standard deviation of 1000 raw images. With the high contrast apparent between the channel and background, the edge of the channel walls can be detected using an image processing algorithm in MATLAB. Figure 3(c) represents the final binarized mask of the geometry generated from the raw images. Having a proper masked geometry allows only the region containing the particle data to be identified as in Figure 3(d) which is for $R e=600$. The final images, similar to the processed image in Figure 3(d), are used for both PTV and PTV combined with the tessellation meshing method.

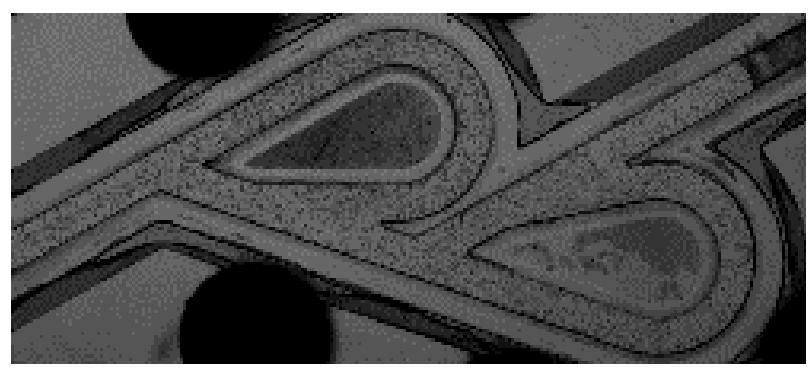

(a)

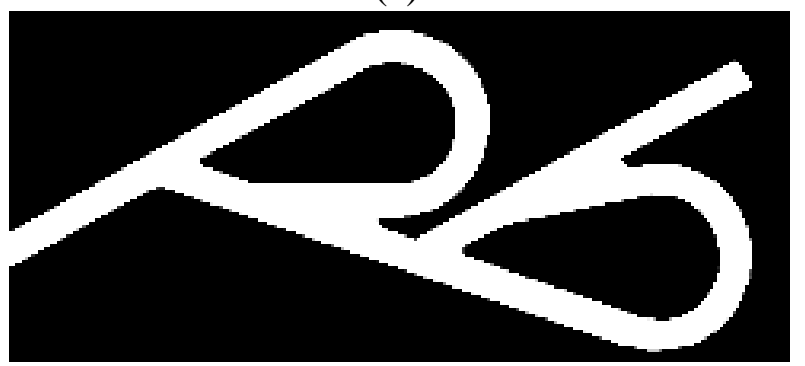

(c)

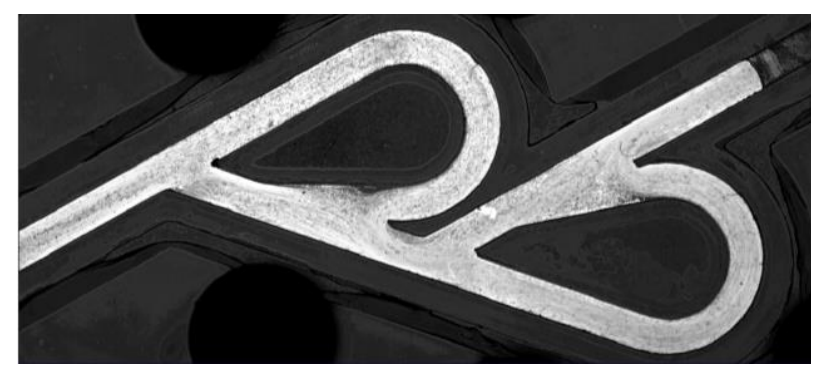

(b)

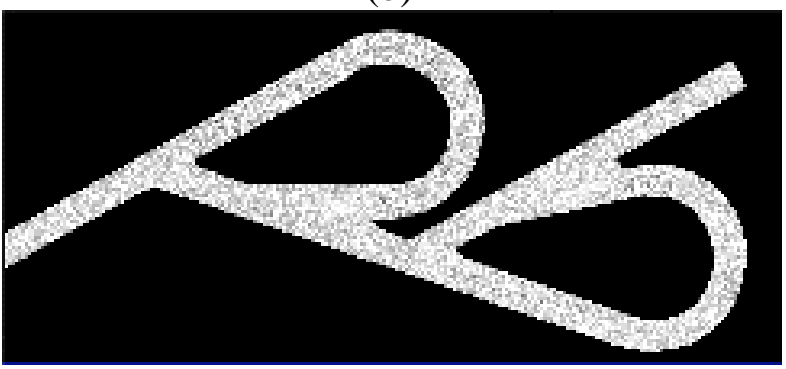

(d)

Figure 3: Image processing steps to obtain the wall boundaries of the geometry; (a) raw image showing the initial domain, (b) standard deviation of the intensities calculated from 1000 raw images, (c) the masked generated by image processing of the standard deviation of 1000 images, (d) result of masked image with seeding particles

\section{Tessellation Combined with PTV}

Preliminary results obtained from PIV and PTV are compared to highlight the difference in the determined near wall flow. Figure 4(a) displays the result of the flow processing using PIV. Vortices can be observed as a result of the flow separation and are also shown as zoomed-in streamlines. Such vortices are observed near the merging and dividing areas where the flow is experiencing a negative pressure 
gradient. The same trend can be observed in Figure 4(b) where PTV is used to process the flow images. A rectangular grid is used to interpolate the velocity distribution in the flow domain calculated using the PTV approach. Comparing the highlighted areas in Figure 4, a major disagreement in the streamlines can be observed close to the wall region which is mainly due to the grid type used for calculating the velocity from PTV data. This highlights that the PIV and PTV approaches should be used carefully in flow studies to ensure the proper interpretation of the flow physics.

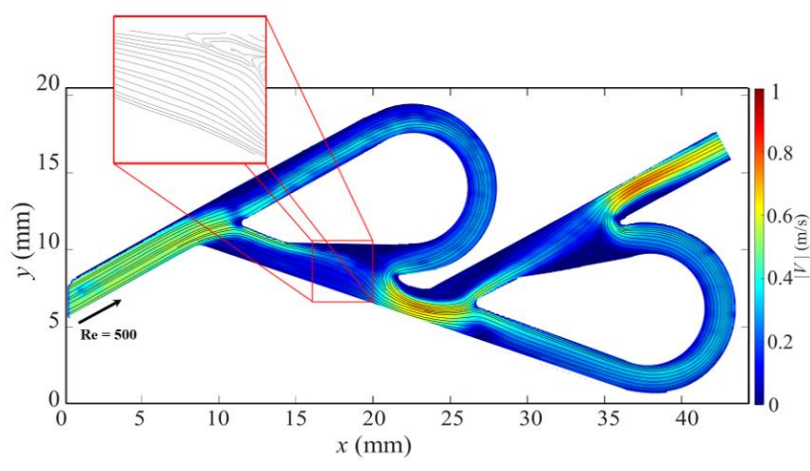

(a)

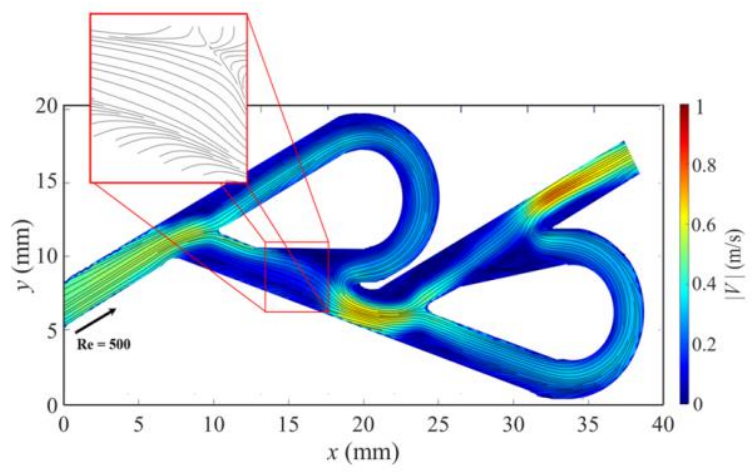

(b)

Figure 4: The velocity field and streamlines of a reverse flow inside the Tesla valve for $\mathrm{Re}=500$ using (a) PIV and (b) PTV method. The highlighted area shows the near wall streamlines

Important flow parameters such as pressure can be calculated after distributing the dispersed PTV velocity vectors, on a discretized domain Azadi et al. (2021). The structured rectangular grids are mostly used for simple geometries. In cases with complex geometries, moving boundaries, or high curved regions such as the Tesla valve geometry, generating a high-quality structured grid becomes more challenging Azadi et al. (2021). To overcome this issue, a tessellation meshing method can be used. This method maps the dispersed PTV velocity data onto an optimized high-quality triangular node structure Azadi et al. (2021).

The mapping process starts with identifying the boundaries of the domain Azadi et al. (2021). As the second step, a sufficient number of nodes is distributed on the boundaries. The tessellated domain forms from the inserted nodes and the specifications of the mesh depending on each case Azadi et al. (2021). The Delaunay triangulation algorithm used in this method, adjusts itself to the perfect position in order to make a high-quality mesh Azadi et al. (2021). This adjustability results in generating a mesh which can be easily matched to the boundaries of the domain. As a result, a precise near wall flow can be determined using the dispersed velocity data from PTV. Figure 5(a) shows the non-uniform vectors calculated from PTV for the forward flow condition for $\mathrm{Re}=100$. The uniformly distributed velocity vectors and the velocity contour which are obtained from the tessellation method are shown in Figure 5(b). 


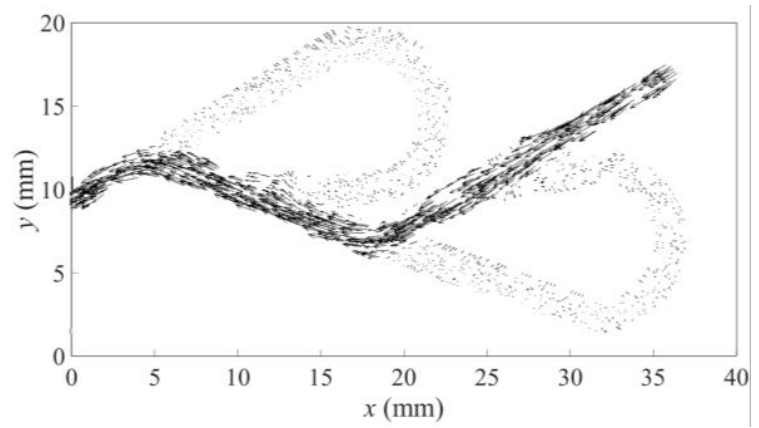

(a)

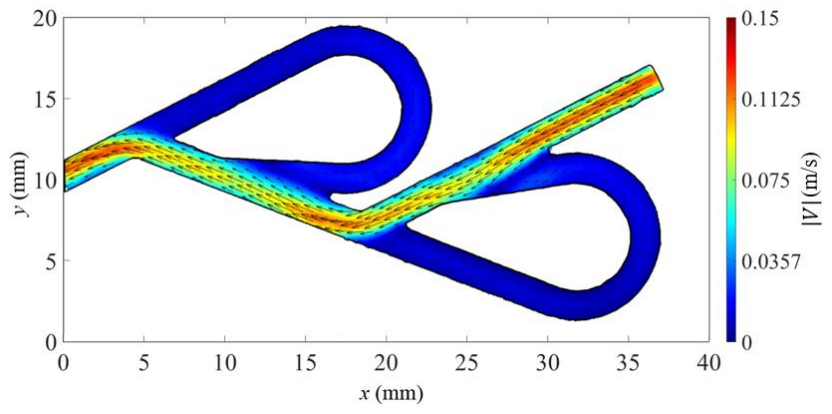

(b)

Figure 5: (a) Non-uniform scattered PTV velocity vectors for the forward flow $\operatorname{Re}=600$, (b) velocity field and uniform vectors after applying the tessellation for the forward flow $\operatorname{Re}=100$, $|V|$ represents the velocity magnitude in the plot.

Three different mesh densities were used to study the effect of the mesh size on the tessellation results. Table 1 contains the properties for each of these cases. It is noticeable that for each case the skewness is less than the recommended amount of 0.33 Azadi et al. (2021). The Tesla valve is made from multiple narrow channels and therefore, to generate a semi-uniform mesh maximum $h_{\max }$ and minimum $h_{\min }$ cell sizes are chosen such that $h_{\max } \leq 2 h_{\min }$. The minimum cell size, $h_{\min }$ is used to distribute nodes on the boundaries and $h_{\max }$ dictates the maximum size for the cells further from the walls.

Table 1 Mesh properties for three different mesh densities.

\begin{tabular}{|c|c|c|c|}
\hline Case & $h_{\min }(\mathrm{mm})$ & $h_{\max }(\mathrm{mm})$ & Skewness \\
\hline 1 & 0.08 & 0.1 & 0.325 \\
\hline 2 & 0.1 & 0.2 & 0.3104 \\
\hline 3 & 0.3 & 0.5 & 0.308 \\
\hline
\end{tabular}

The tessellation results for all 3 grids are investigated to find the optimum mesh size, and the results are shown in Figure 6. The velocity field of the reverse flow condition with $\mathrm{Re}=100$ is chosen and shown for all cases in Figure 6(a). From the highlighted areas it can be realized that all of the cases represent the same velocity gradient. It is also notable that as the mesh becomes smaller, more details are provided by the tessellation. Figure 6(b) represents the velocity profile for a certain location $x=32 \pm 0.05 \mathrm{~mm}$. The velocity profiles for all cases start from zero at the walls, reach the maximum velocity and follow the same trend. Case 3, the largest mesh size, diverges from the trend in some points which means that in some locations some of the velocity data points are neglected. On the other hand the profile for Case 1 , the smallest mesh, fluctuates in the region highlighted in Figure 6(b). This fluctuation is an indicator for the lack of information. This means that the distance between dispersed values extracted from the PTV is too large and the velocity value is only the output of an interpolation regardless of the small mesh size. It can be concluded that for this data set, Case 2 is an appropriate mesh size which provides a reliable velocity profile based on the PTV data. 


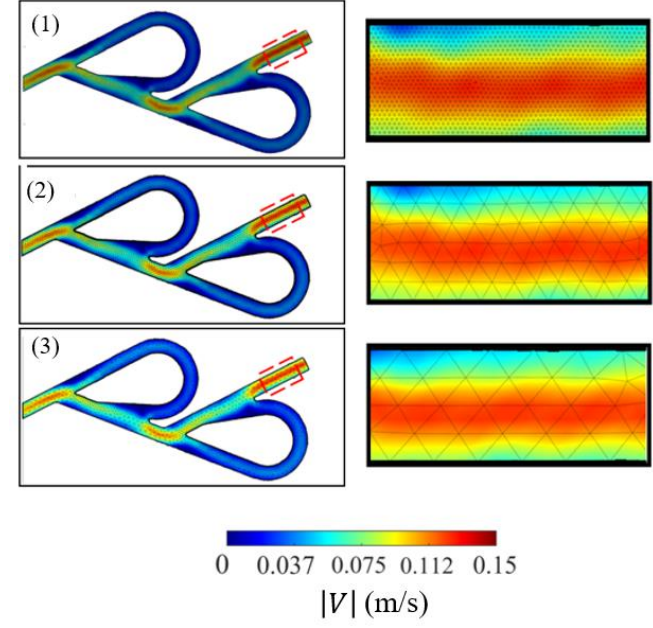

(a)

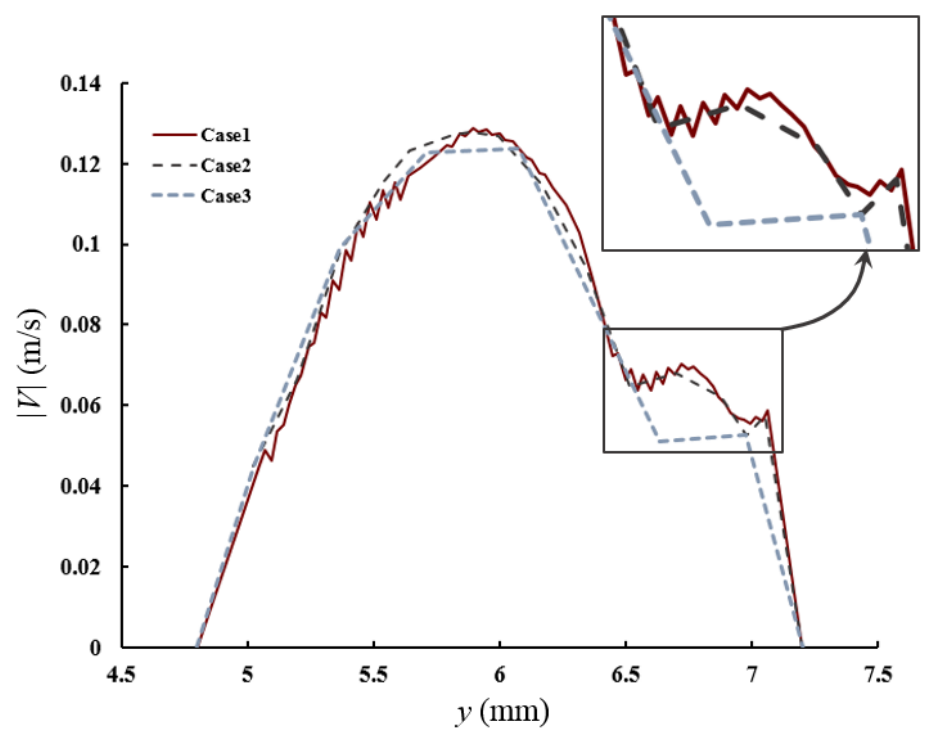

(b)

Figure 6: (a) Representation of the mesh generated effect on the velocity field for 3 different mesh densities in Tesla valve, (b) the comparison of the velocity profile calculated for these cases.

\section{Results and discussion}

This section discusses the results obtained using different techniques including standard PIV, standard PTV, tessellated PTV, and PTV interpolated onto a structured grid. The discussion is mainly focused on the near wall velocity vectors where the difference between each technique is significantly distinguishable.

Near wall vectors for forward flow with $\mathrm{Re}=600$ are shown in Figure 7 comparing the effect of a structured grid and a tessellated grid on the velocity field generated using the PTV method. Velocity vectors near the wall in Figure 7(a) are pointing toward the walls when there is no tessellation. This means that a velocity component normal to the wall is added to the flow. The normal component indicates that flow passes through the solid channel walls contrasting the physics. Figure 7(b) shows the same field of view with the velocity field discretized to the unstructured mesh using the tessellation method. Unlike the velocity field from the structured mesh in Figure 7(a), velocity components normal to the wall are eliminated in Figure 7(b). Furthermore, interpolated PTV data in Figure 7(a) is not perfectly aligned with the channel wall. This misalignment generates extra small triangular regions outside the channel. These additional regions are corrected using the triangular grid generated and perfectly adjusted by tessellation. 
(a)

(b)

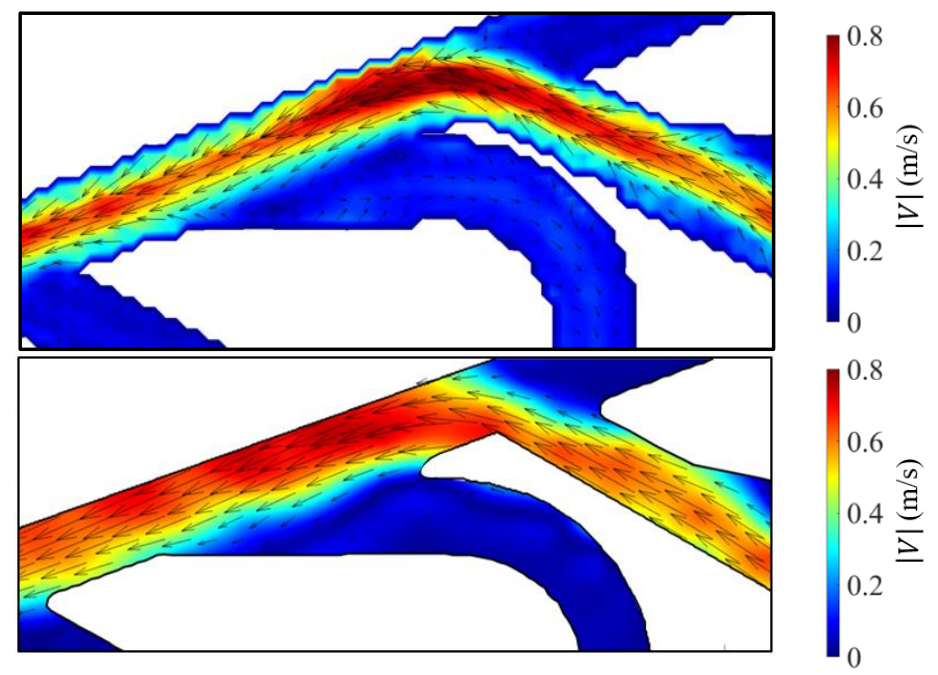

Figure 7: (a) The velocity vectors coming out of the wall in forward flow at $\mathrm{Re}=600$ using PTV, (b) near wall treatment of the PTV obtained velocity vectors using tessellation algorithm at

$$
\operatorname{Re}=600
$$

The effectiveness of the tessellation on dispersed PTV results is compared to disperse PTV, PTV interpolated on the structured grid, and PIV results is shown in Figure 8. Velocity is extracted from a $1 \mathrm{~mm}$ range on the $x$-axis $4.5 \mathrm{~mm} \leq x \leq 5.5 \mathrm{~mm}$ as shown in the top right corner of Figure 8 on the geometry. This is to ensure sufficient data points are collected from all methods. Figure 8 shows that all of the methods follow the same trend. In Figure 8 regions (I) and (II) show that dispersed PTV and interpolated PTV on the structured grid data do not indicate the near wall velocity values. Despite having a similar trend, as it can be seen from Figure 8, no velocity data is obtained near the wall from different techniques except for the tessellated PTV. Tessellated PTV results shows a similar but smoother trend to PIV results shown in Figure 8. It is also observable in Figure 8 regions (I) and (II) that tessellated PTV provides the near wall results with velocity profile and trend similar to PIV results.

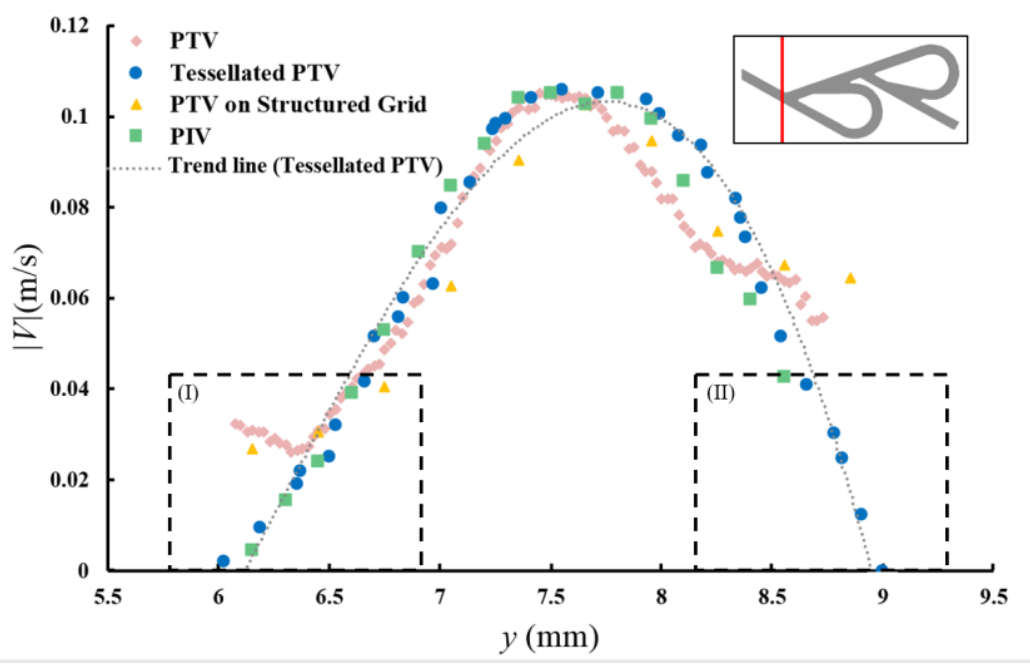

Figure 8: (a) Velocity magnitude for data points $4.5 \mathrm{~mm} \leq x \leq 5.5 \mathrm{~mm}$ calculated by PIV and PTV distributed with structured and triangular mesh for Tesla with reverse flow and $\operatorname{Re}=100$ and the trend line to the tessellated PTV data; regions (I) and (II) highlights the near wall areas 
Using the tessellated PTV approach, the effect of flow rate and flow direction are explored for the valve. The velocity field with uniform flow velocity for $\mathrm{Re}=100$ is shown in Figure 10 for forward and reverse flows. Flow velocity near the wall is corrected using the tessellation method. The vortices in the reverse direction are observable in Figure 10(a) which indicates that tessellation can distribute velocity without eliminating the vortices. Figure 10(b) shows the flow in the forward direction where flow passes the Tesla valve with maximum flow rate. As it is seen from Figure 10(b) the flow in the branches in forward direction is almost stationary.

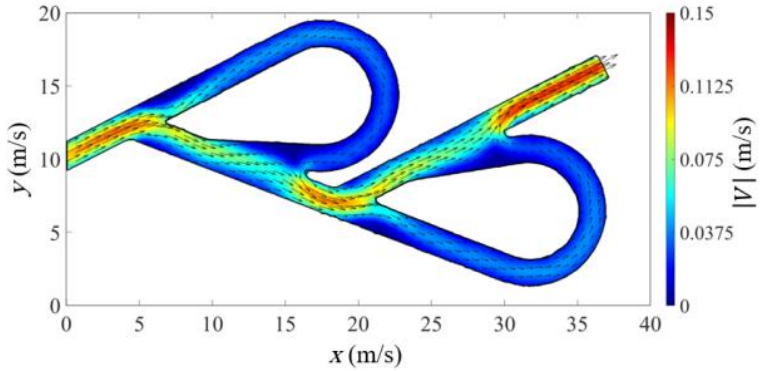

(a)

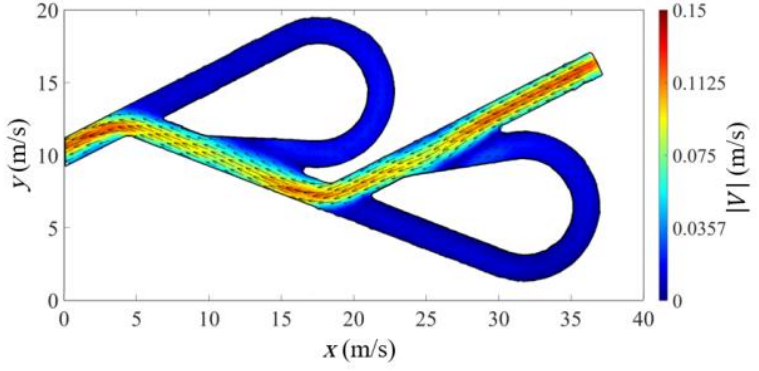

(b)

Figure 9: Tessellated velocity field and uniform velocity vector distribution for flow at $\operatorname{Re}=100$ in (a) reverse and (b) forward direction

With increasing the Reynolds number to 600 vortices start to grow more in areas where the flow is separated. As a result, the changes in the velocity value and components increases in all directions. This makes the velocity transportation from a dispersed state to the tessellated map more challenging. As shown in Figure 10, velocity direction is treated using the tessellation method near the wall. For both flow directions shown in Figure 10(a) and (b), a smooth velocity field is achieved. As shown in Figure 10(a) flow in a reverse direction generates vortices which are responsible for the pressure drop and the reduction of the outlet flow rate. In Figure 10(b) forward flow is presented in which the flow is passing through the Tesla valve with minimum detaching on the loops joint. As a result, in the forward direction the flow inside the loops is almost stationary whereas in the reverse direction the flow stream is clearly detected inside the loops.

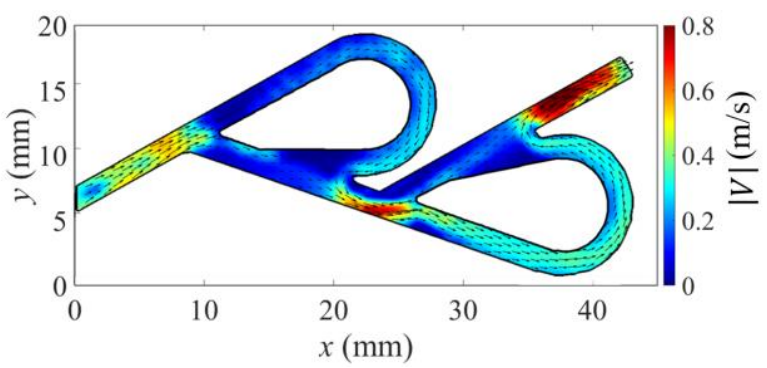

(a)

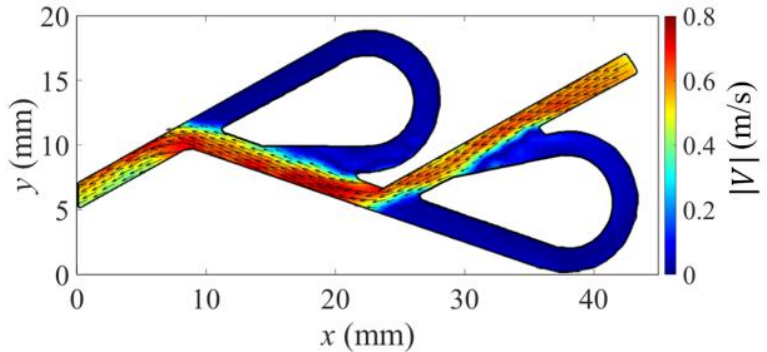

(b)

Figure 10: Tessellated velocity field and uniform velocity vector distribution for flow at $\operatorname{Re}=600$ in (a) reverse and (b) forward direction 


\section{Conclusion}

A meshing methodology to apply tessellation in a complex geometry such as a multi-section Tesla valve is investigated. Four different approaches are compared to obtain the velocity profile including standard PIV, standard PTV, tessellated PTV and PTV interpolated onto a structured grid. The results shows tessellated PTV provides more details and smoother results in certain regions where other techniques miss the data. The results also indicate that the tessellation method can be a reliable method for discretizing the dispersed results for complex geometries. Since this method provides access to boundaries of the channel to set boundary conditions, the velocity field generated by the tessellation method can be used for calculating pressure.

\section{References}

Azadi, Reza, Jaime Wong, and David S. Nobes. (2021) Determination of fluid flow adjacent to a gas/liquid interface using particle tracking velocimetry (PTV) and a high-quality tessellation approach. Experiments in Fluids 62: 1-20.

Gamboa, Adrian R., Christopher J. Morris, and Fred K. Forster. (2005) Improvements in fixed-valve micropump performance through shape optimization of valves. Journal of Fluids Engineering, Transactions of the ASME 127: 339-46.

Porwal, Piyush R., Scott M. Thompson, D. Keith Walters, and Tausif Jamal. (2018) Heat Transfer and Fluid Flow Characteristics in Multistaged Tesla Valves. Numerical Heat Transfer; Part A: Applications 73: 347-65.

Raffel, Jan. (2019). Influence of the Reynolds number on the stationarity of the flow field in a Tesla diode a bachelor thesis. Leibniz University Hannover.

Raffel, Jan, Shadi Ansari, and David S. Nobes. (2021) An experimental investigation of flow phenomena in a multi-stage micro tesla valve. Journal of Fluids Engineering, 21-1079

Raffel, Markus, Christian E Willert, Fulvio Scarano, and Christian J Kähler. 2007. Particle Image Velocimetry a Practical Guide. Germany.

Tesla, Nikola. 1920. Patented Feb. 3, 1920., issued 1920.

Wahidi, Tabish, Rajat Arunachala Chandavar, and Ajay Kumar Yadav. (2020) Stability enhancement of supercritical $\mathrm{CO}_{2}$ based natural circulation loop using a modified Tesla valve." Journal of Supercritical Fluids 166: 105020.

Ansari, Shadi, Michael Bayans, Faezeh Rasimarzabadi, and David S Nobes. (2018) Flow visualization of the Newtonian and non- Newtonian behavior of fluids in a Tesla-diode valve. 5th International Conference on Experimental Fluid Mechanics - ICEFM 2018 Munich, Munich, Germany. 